\title{
Skorohod's representation theorem and optimal strategies for markets with frictions
}

\author{
Huy N. Chau* Miklós Rásonyi*
}

April 13, 2017

\begin{abstract}
We prove the existence of optimal strategies for agents with $\mathrm{cu}-$ mulative prospect theory preferences who trade in a continuous-time illiquid market, transcending known results which pertained only to risk-averse utility maximizers. The arguments exploit an extension of Skorohod's representation theorem for tight sequences of probability measures. This method is applicable in a number of similar optimization problems.
\end{abstract}

Keywords: Optimization, non-concave utility, Skorohod's representation, illiquidity, market frictions.

\section{Introduction}

Optimal investment for an agent with given preferences has always been a core topic in mathematical finance. Classical papers on the subject $([36,52])$ as well as most subsequent studies neglected the presence of market frictions such as transaction costs, taxes and liquidity effects, and they also stuck to the paradigm of a concave utility function expressing risk-aversion of the agent.

Non-concave preferences involving distorted probabilities emerged over the time, [32, 43, 54], and incorporating frictions in the model led to mathematical settings that are different from the classical one, see e.g. [1] and Chapter 3 of [30].

*Alfréd Rényi Institute of Mathematics, Hungary Hungarian Academy of Sciences, Reáltanoda utca 13-15, Budapest, Hungary (chau@renyi.hu, rasonyi@renyi.hu). 
Our purpose in the present paper is to prove existence theorems for optimal strategies in a general, continuous-time setting, following the footsteps of [35, 31, 53, 6, 17, 39, 10, 12, 19, 41]. Just like [10, 12, 19, 41], we wish to treat markets with friction. The essential novelty is that our method allows preferences that correspond to possibly non-concave utility functions and may involve distorted probabilities. Such problems seem to be intractable with the usual techniques of convex duality and arguments involving convex combinations, [35, 31, 53]. We propose a method for establishing the existence of optimizers based on an extension of Skorohod's famous representation theorem, see Theorem 2.1 and Remark 4.7 below.

The approach we present works for preferences of a very general form and for various financial models. Here we confine ourselves to the illiquid market of [22] and to preferences in the spirit of cumulative prospect theory (CPT), see [32, 54]; this setting illustrates the power of the method fairly well. Further extensions are left for future research. We also point out that our method seems flexible enough for applications to e.g. model uncertainty where expected utility is maximized in the worst-case sense over a set of probabilities, see Remark 4.10 below.

Optimal investment with CPT preferences concentrated almost exclusively on frictionless markets: [5, 25] treated one-step models and found rather precise conditions for the existence of optimal portfolio. The papers [14, 47] considered multistep models and proved that there are optimal strategies when the investor is allowed to use a randomization (which is independent of the market). The present paper is similar to [14, 47] in the sense that we also allow randomization, see Assumption 3.2 below.

Most continuous-time studies assumed a complete market: [4, 13, 50] considered nonconcave utilities but no probability distortions; in [27] explicit solutions were obtained under suitable assumptions, see also [15]; [11] considered informational aspects of the problem while [45, 46] investigated well-posedness. Only a narrow class of incomplete markets have been treated so far, [51, 45, 48], using ad hoc techniques. Further problems of optimal control within CPT were treated in [28, 16, 24, 23] but these are connected to our setting only remotely.

We are aware of only [40] that treats markets with frictions and agents with nonconcave preferences. That paper established a fairly general dynamic programming principle in a discrete time setting without probability distortions which is applicable to optimization problems in a wide range of market models. The present paper seems to be the first continuous-time study involving CPT preferences and market frictions at the same time.

In Section 2 we present an extension of Skorohod's representation theorem from [26] and verify that it applies to our setting. In Section 3 we present a 
model of an illiquid market. In Section 4 we use the representation theorem of Section 2 to construct optimal strategies in investment problems under liquidity constraints. Section 5 sketches an alternative formulation for our results. Finally, Section 6 collects some useful lemmas.

\section{A representation theorem}

For a random variable $X$ on some probability space we denote by $\operatorname{Law}(X)$ its law. When there might be an ambiguity about the probability space we use the notation $\operatorname{Law}_{Q}(X)$ for the law of $X$ under the probability $Q$.

We denote by $\mathcal{B}(Z)$ the Borel-field of a topological space $Z$. A sequence of probabilities $\mu_{k}, k \in \mathbb{N}$ on $\mathcal{B}(Z)$ is said to be tight if, for all $\varepsilon>0$, there is a compact $K(\varepsilon) \subset Z$ such that, for all $k, \mu_{k}(Z \backslash K(\varepsilon))<\varepsilon$. We first recall a remarkable result from [26].

Theorem 2.1. Let $Z$ be a topological space such that there is a countable collection $f_{i}, i \in \mathbb{N}$ of continuous, real-valued functions which separate points on $Z$. Let $\mu_{k}, k \in \mathbb{N}$ be a tight sequence of measures on $\mathcal{B}(Z)$. Then there is a subsequence $k_{j}, j \in \mathbb{N}$ and a probability space on which there exist $Z$-valued random variables $\xi, \xi_{j}$, with $\operatorname{Law}\left(\xi_{j}\right)=\mu_{k_{j}}, j \in \mathbb{N}$ and $\xi_{j} \rightarrow \xi$ a.s., $j \rightarrow \infty$.

Lemma 2.2. Let $Z$ be a regular Hausdorff topological space such that there is an increasing sequence $A_{n}, n \in \mathbb{N}$ of closed subspaces of $Z$ which are separable metric spaces (under a suitable metric) and $Z=\cup_{n \in \mathbb{N}} A_{n}$. Then there is a countable collection of continuous, real-valued functions which separate points on $Z$.

Proof. Each $A_{n}$ is Lindelöf hence so is $Z$. A Lindelöf regular space is normal, so $Z$ is also a normal Hausdorff space. For each $n$, there is clearly a sequence $f_{i}^{n}, i \in \mathbb{N}$ of continuous real-valued functions on $A_{n}$ which separate points on $A_{n}$. These can be extended in a continuous way to $Z$ by Tietze's theorem, for all $n, i$. Then the countable collection of extended functions $f_{i}^{n}, i, n \in \mathbb{N}$ separates points on $Z$.

Corollary 2.3. Let $\mathbb{B}$ be a separable Banach space with dual $\mathbb{B}^{\prime}$ equipped with the weak-star topology and let $M$ be a separable metric space. Then $Z:=\mathbb{B}^{\prime} \times M$ satisfies the hypotheses of Lemma 2.2.

Proof. Indeed, topological vector spaces and metric spaces are both regular; $\mathbb{B}^{\prime}$ as well as $M$ are clearly Hausdorff. So the product $Z$ is regular Hausdorff. Denote by $\|\cdot\|^{\prime}$ the norm of $\mathbb{B}^{\prime}$ and set $B_{n}:=\left\{x \in \mathbb{B}^{\prime}:\|x\|^{\prime} \leq n\right\}$. In the 
weak-star topology, any closed ball in the dual of a separable Banach space is metrizable and compact, so $A_{n}:=B_{n} \times M$ is a separable metric space, closed in the relative topology of $Z$, for each $n$.

Example 2.4. Fix $1<\beta<\infty$. Let $\gamma$ be defined by $1 / \beta+1 / \gamma=1$. Let $L^{\beta}:=L^{\beta}([0,1], \mathcal{B}([0,1]), L e b)$ denote the usual Banach space of (equivalence classes of) $\beta$-integrable functions on the unit interval. Let $T$ be $L^{\beta}$ equipped with the weak topology. $L^{\beta}$ is the dual of the separable Banach space $L^{\gamma}$ and the weak topology on $L^{\beta}$ is precisely the weak-star topology in the duality $\left(L^{\gamma}, L^{\beta}\right)$. For any separable metric space $M$, Theorem 2.1 applies to $Z:=$ $T \times M$, by Lemma 2.2 and Corollary 2.3 ,

This topological space $Z$ will be crucial in our study of optimal investment in illiquid markets as strategies will be represented by random elements in $T$ and a certain $M$ will code the information structure of the market, see Section 3 for details.

Example 2.5. Consider $C[0,1]$, the separable Banach space of continuous functions on the unit interval (with the supremum norm). Let $\mathcal{M}$ denote the Banach space of finite signed measures on $\mathcal{B}([0,1])$, the dual space of $C[0,1]$. We take $T$ to be $\mathcal{M}$ equipped with the weak-star topology. Again, Theorem 2.1 applies to $Z:=T \times M$ for any separable metric space $M$.

The space $Z$ can be used in the treatment of optimal investment under transaction costs where strategies correspond to random elements in $T$ and the price process is assumed continuous (i.e. it is a random element in $C[0,1])$. Due to the numerous technicalities, details will not be presented here.

Remark 2.6. For the moment, the case of frictionless markets is not accessible with our methods as the construction of stochastic integrals is carried out in a filtration-dependent way and cannot be performed pathwise.

Remark 2.7. Note that neither of the spaces in Examples 2.4, 2.5 is metrizable so the well-known versions of Skorohod's representation theorem (see e.g. Lemma 4.30 in [33]) are not applicable to them. We also point out that topological spaces with a Skorohod representation property behave delicately: they are not known to be closed for topological products; counterexamples show that, even for a weakly convergent sequence of probabilities, Skorohod representation may only work for a subsequence, etc. We refer the interested reader to [2] for details. 


\section{A model of an illiquid market}

We now recall a simple version of the market model in [22] where security prices depend on the trading speed. In that model, price impact is assumed instantaneous (the activities of the small agent in consideration do not move prices permanently) and superlinear, see Assumption 3.7 below. Superlinearity is in accordance with empirical studies, see e.g. [18].

We will assume throughout the paper that trading takes place continuously in the time interval $[0,1]$. Let $\left(\Omega, \mathcal{F},\left(\mathcal{F}_{t}\right)_{t \in[0,1]}, P\right)$ be a filtered probability space, where the filtration is complete and right continuous, $\mathcal{F}_{0}$ is trivial. A process $\psi$ on this space is an $\mathcal{F} \otimes \mathcal{B}([0,1])$-measurable mapping on $\Omega \times[0,1]$. The notation $E X$ will refer to the expectation of the random variable $X$. If there is ambiguity about the probability space then $E_{Q} X$ will denote the expectation of $X$ under the probability $Q$. We denote by $1_{A}$ the indicator of a set $A$.

In the sequel we will need that the filtration is of a specific type and that the probability space is large enough.

Assumption 3.1. There exists a càdlàg $\mathbb{R}^{m}$-valued process $Y$ with independent increments such that $\mathcal{F}_{t}$ is the $P$-completion of $\sigma\left(Y_{u}, 0 \leq u \leq t\right)$, for $t \in[0,1]$.

Assumption 3.2. There exists a random variable $U$ that is uniformly distributed on $[0,1]$ and independent of $\mathcal{F}_{1}$.

For $m \in \mathbb{N}$, we denote by $\mathcal{D}^{m}$ the space of $\mathbb{R}^{m}$-valued right-continuous functions with left-hand limits on [0,1], equipped with Skorohod's topology, see Chapter 3 of [8].

Remark 3.3. The Borel-field of $\mathcal{D}^{m}$ is generated by the coordinate mappings $x \in \mathcal{D}^{m} \rightarrow x(t) \in \mathbb{R}^{m}, t \in[0,1]$, see Theorem 12.5 of [8]. It follows that the function $\omega \in \Omega \rightarrow Y(\omega) \in \mathcal{D}^{m}$ is a random variable and so is $\omega \in$ $\Omega \rightarrow{ }^{t} Y(\omega) \in \mathcal{D}^{m}$, for all $t \in[0,1]$, where ${ }^{t} Y$ is the process defined as $\left({ }^{t} Y\right)_{u}=Y_{u} 1_{[0, t)}+Y_{t} 1_{[t, 1]}, u \in[0,1]$. Furthermore, $\mathcal{F}_{t}=\sigma\left(Y_{s}, s \leq t\right)=\sigma\left({ }^{t} Y\right)$, for all $t \in[0,1]$.

Let us define the augmented filtration $\mathcal{G}_{t}:=\mathcal{F}_{t} \vee \sigma(U)$. Standard arguments (like Lemma 4.9 of [49]) imply that $\mathcal{G}_{t}, t \in[0,1]$ also satisfies the usual hypotheses of completeness and right-continuity.

The market consists of a riskless asset $S^{0}$ with price $S_{t}^{0}=1$ for all $t \in[0,1]$ ("money account") and a risky asset whose price $S$ is assumed to be an $\mathbb{R}$ valued càdlàg adapted process. (The extension of our results is straightforward to the case of multiple risky assets.) 
Lemma 3.4. There exists a measurable function $f: \mathcal{D}^{m} \rightarrow \mathcal{D}^{1}$ such that $S=f(Y)$. Furthermore, ${ }^{t} S$ is measurable with respect to $\sigma\left({ }^{t} Y\right)$, for all $t \in[0,1]$, where ${ }^{t} S$ is the process defined as $\left({ }^{t} S\right)_{u}=S_{u} 1_{[0, t)}+S_{t} 1_{[t, 1]}, u \in[0,1]$.

Proof. As $S$ is a càdlàg process, it is a $\mathcal{D}^{1}$-valued random variable, by the argument of Remark 3.3. The first statement now follows from Doob's lemma (Lemma 1.13 of [33]) since $S$ is $\sigma(Y)$-measurable. The second statement also follows as in Remark 3.3 .

Definition 3.5. A feasible strategy is a process $\phi: \Omega \times \mathbb{R}_{+} \rightarrow \mathbb{R}$ such that it is progressively measurable with respect to $\mathcal{G}_{t}, t \in[0,1]$ and

$$
\int_{0}^{1}\left|\phi_{t}\right| d t<+\infty, \text { a.s. }
$$

$W e$ denote by $\mathcal{A}$ the set of all feasible strategies.

Remark 3.6. We indicate that Definition 3.5 slightly deviates from the corresponding Definition 2.1 in [22]. In that paper strategies are assumed optional while here we only require progressive measurability. The latter class fits better the purposes of the present paper and the proofs of all the results we cite from [22] (Lemma 3.4 and Theorem 5.1) go through without any modifications for the class of progressively measurable processes as well.

The process $\phi$ represents the trading rate. Assume that the initial positions in the money account and in the stock are $z^{0}, z^{1}$, respectively. For each $\phi \in \mathcal{A}$, we may define by

$$
\varphi_{t}:=z^{1}+\int_{0}^{t} \phi_{u} d u, \quad t \in[0,1]
$$

the number of risky assets in the portfolio at time $t$.

If there were no liquidity effects, the self-financing condition would imply that the change of the portfolio value over $[0, t]$ is $\int_{0}^{t} \varphi_{u} d S_{u}$ (implicitly assuming that $S$ is a semimartingale). As the value of the stock position at $t$ is $\varphi_{t} S_{t}$, a heuristic integration by parts gives that the value at $t$ of the money account is

$$
\begin{aligned}
z^{0}+\int_{0}^{t} \varphi_{u} d S_{u}-\phi_{t} S_{t} & = \\
z^{0}-\int_{0}^{t} S_{u} d \varphi_{u} & =z^{0}-\int_{0}^{t} \phi_{u} S_{u} d u .
\end{aligned}
$$

Notice that the last expression makes mathematical sense for any $\phi \in \mathcal{A}$ and for any càdlàg $S$. 
We now add liquidity effects to our model by a function $G$ in such a way that $G_{t}(x)$ represents the "penalty" for trading at speed $x$ at time $t$.

Assumption 3.7. There is $\alpha>1$ and a continuous function $H: \mathbb{R} \rightarrow \mathbb{R}$, such that $G_{t}(x)=g\left(S_{t}, x\right)$ with

$$
g(s, x)=H(s)|x|^{\alpha}
$$

and $\inf _{t \in[0, T]} H\left(S_{t}\right)>0$ a.s. Furthermore, fix $1<\beta<\alpha$ and assume

$$
E \int_{0}^{1} H^{\beta /(\beta-\alpha)}\left(S_{t}\right)\left(1+\left|S_{t}\right|\right)^{\beta \alpha /(\alpha-\beta)} d t<\infty .
$$

Remark 3.8. Typical specifications are $G_{t}(x)=\lambda|x|^{\alpha}$ or $G_{t}(x)=\lambda S_{t}|x|^{\alpha}$ with some $\alpha>1, \lambda>0$, see e.g. [20]. The first one satisfies Assumption 3.7 whenever $\int_{0}^{T} E\left|S_{t}\right|^{\beta \alpha /(\alpha-\beta)} d t<\infty$, the second one whenever $S$ is positive, has continuous trajectories and $\int_{0}^{T}\left[E\left|S_{t}\right|^{\beta(\alpha-1) /(\alpha-\beta)}+E\left|S_{t}\right|^{-\beta /(\alpha-\beta)}\right] d t<\infty$. It would be possible to substantially relax both (21) and (3) and to allow dependence of $H$ on the whole trajectory of $S$ but this would lead to complications without enhancing the message of our paper, so we refrain from seeking greater generality.

Definition 3.9. For a given strategy $\phi \in \mathcal{A}$ and an initial position $z \in \mathbb{R}^{2}$, the positions at time $t \in[0, T]$ in the risky and riskless asset are defined as

$$
\begin{aligned}
& \tilde{X}_{t}(\phi):=z^{1}+\int_{0}^{t} \phi_{u} d u \\
& X_{t}(\phi):=z^{0}-\int_{0}^{t} \phi_{u} S_{u} d u-\int_{0}^{t} G_{u}\left(\phi_{u}\right) d u,
\end{aligned}
$$

respectively (compare to (11) above).

Note that $X_{t}(\phi)$ may take the value $-\infty$. For simplicity, we assume from now on that $z^{0}=z^{1}=0$, the case of nonzero initial positions is easily incorporated into the present setting.

Let $G^{*}$ be the Fenchel-Legendre conjugate of $G$,

$$
G_{t}^{*}(y):=\sup _{x \in \mathbb{R}}\left(x y-G_{t}(x)\right)=\frac{\alpha-1}{\alpha} \alpha^{1 /(1-\alpha)} H^{1 /(1-\alpha)}\left(S_{t}\right)|y|^{\alpha /(\alpha-1)},
$$

as an elementary calculation shows. From (4), under Assumption 3.7 one has

$$
X_{1}(\phi) \leq B:=\int_{0}^{1} G_{t}^{*}\left(-S_{t}\right) d t<\infty, \text { a.s. }
$$

see Lemma 3.1 of [22]. We call $B$ the market bound as it dominates the terminal money account position of any feasible portfolio. 


\section{Optimal investments}

For $x \in \mathbb{R}$ we denote $x^{+}:=\max \{x, 0\}, x^{-}:=\max \{-x, 0\}$. Let $u_{+}, u_{-}$: $\mathbb{R}_{+} \rightarrow \mathbb{R}_{+}$be continuous, increasing functions such that $u_{ \pm}(0)=0$. Let $w_{+}, w_{-}:[0,1] \rightarrow[0,1]$ be continuous with $w_{ \pm}(0)=0, w_{ \pm}(1)=1$. Functions $u_{ \pm}$express the agent's attitude towards gains and losses while $w_{ \pm}$are functions distorting the probabilities of events, see [54], [14].

We define, for any random variable $X \geq 0$,

$$
V_{+}(X):=\int_{0}^{\infty} w_{+}\left(P\left(u_{+}(X) \geq y\right)\right) d y
$$

and

$$
V_{-}(X):=\int_{0}^{\infty} w_{-}\left(P\left(u_{-}(X) \geq y\right)\right) d y
$$

For each real-valued random variable $X$ with $V_{-}\left(X^{-}\right)<\infty$ we set

$$
V(X):=V_{+}\left(X^{+}\right)-V_{-}\left(X^{-}\right) .
$$

Let $W$ be an $\mathcal{F}_{1}$-measurable random variable representing a benchmark for the agent in consideration. For example, $W$ can be the value of an index or of the portfolio of a rival at time 1 which serves as a reference point for our investor. The quantity $V(X-W)$ expresses the satisfaction of an agent with $\mathrm{CPT}$ preferences when (s)he receives a random amount $X$, see [27, 14] for more detailed discussions. Positive $X-W$ means outperforming a benchmark, negative $X-W$ means falling short of it. Doob's theorem implies that there is a measurable $\ell: \mathcal{D}^{m} \rightarrow \mathbb{R}$ such that $W=\ell(Y)$.

Let us define $\mathcal{A}^{\prime}:=\left\{\phi \in \mathcal{A}: \tilde{X}_{1}=0, V_{-}\left(\left[X_{1}(\phi)-W\right]^{-}\right)<\infty\right\}$. For each $\phi \in \mathcal{A}^{\prime}$ the position in the risky asset is liquidated by the terminal date 1 and the utility functional $V$ is well-defined for the value of the money account at 1 minus the benchmark. We aim to find an optimal investment strategy, i.e. $\phi^{\dagger} \in \mathcal{A}^{\prime}$ with

$$
V\left(X_{1}\left(\phi^{\dagger}\right)-W\right)=\sup _{\phi \in \mathcal{A}^{\prime}} V\left(X_{1}(\phi)-W\right) .
$$

Remark 4.1. Note that if $w_{ \pm}(p)=p$ (that is, there is no distortion) then we have $V(X)=E u(X)$ where $u(x)=u_{+}(x), x \geq 0$ and $u(x)=-u_{-}(-x)$ for $x<0$. This shows that the above setting generalizes the well-known expected utility framework, see e.g. [35, 31, 53, 6, 7].

It would be possible to prove analogues of Theorem 4.5 below for other types of objectives e.g. the performance measures of [17]. We stress that the main purpose of the present paper is to demonstrate a useful method and not to explore all possible ramifications. 
Assumption 4.2. We assume that $V_{+}\left([B-W]^{+}\right)<\infty$ and $E W^{+}<\infty$. Furthermore, there exist $0<\delta_{2}<\delta_{1}$ such that

$$
u_{-}(x) \geq c_{1} x^{\delta_{1}}-c_{2}
$$

and

$$
w_{-}(p) \geq c_{3} p^{\delta_{2}}
$$

with some constants $c_{1}, c_{2}, c_{3}>0$ and for all $x \in \mathbb{R}_{+}, p \in[0,1]$.

Remark 4.3. $V_{+}\left([B-W]^{+}\right)<\infty, E W^{+}<\infty$ are integrability conditions that are easy to verify in concrete situations. Specifications of $u_{-}, w_{-}$satisfy (77), (8) with some $\delta_{1}, \delta_{2}>0$ quite often, going back to [54]. It was shown in [45] that in a frictionless Black-Scholes market $\delta_{1}>\delta_{2}$ is necessary for well-posedness of (44). Hence the conditions of Assumption 4.2 are rather natural. If we assumed $u_{+}$bounded above, we could substantially relax (7) and (8) along the lines of [46].

For comparisons with Theorem 4.5 below, we recall a consequence of Theorem 5.1 in [22].

Theorem 4.4. Let Assumption 3.7 be in vigour, let $u: \mathbb{R} \rightarrow \mathbb{R}$ be concave and nondecreasing, and let $E|u(B-W)|<\infty$ hold. If $\mathcal{A}^{\circ} \neq \emptyset$ then there is $\phi^{\dagger} \in \mathcal{A}^{\circ}$ such that

$$
E u\left(X_{1}\left(\phi^{\dagger}\right)-W\right)=\sup _{\phi \in \mathcal{A}^{\circ}} E u\left(X_{1}(\phi)-W\right),
$$

where $\mathcal{A}^{\circ}=\left\{\phi \in \mathcal{A}: \tilde{X}_{1}(\phi)=0, E\left(u\left(X_{1}(\phi)\right)\right)^{-}<\infty\right\}$.

Proof. Assumptions 2.2 and 2.3 of [22] hold by Assumption 3.7 above hence Theorem 5.1 of 22 applies. We remark that, regrettably, the condition $\mathcal{A}^{\circ} \neq \emptyset$ is missing from the statement of Theorem 5.1 of [22] though it is clearly necessary. Here we publish a corrected statement.

The next theorem is the main result of the present paper which extends Theorem 4.4 to a much broader family of preferences.

Theorem 4.5. Let Assumptions [3.1, 3.2, 3.7 and 4.2 be in vigour. If $\mathcal{A}^{\prime} \neq \emptyset$ then there exists $\phi^{\dagger} \in \mathcal{A}^{\prime}$ such that

$$
V\left(X_{1}\left(\phi^{\dagger}\right)-W\right)=\sup _{\phi \in \mathcal{A}^{\prime}} V\left(X_{1}(\phi)-W\right) .
$$


Proof. We provide a quick overview of the main steps in our argument. Taking an optimizer sequence we show that their laws form a tight sequence (on $L^{\beta}$ with the weak topology). Then we invoke results of Section 2 to realize (on another probability space) a sequence whose members have the same laws but which converge almost surely. Using convex combinations coming from the theorem of Komlós we can show that the limit is an optimizer. However, we have to construct this optimizer on the original space as well so we use $U$ and rely on the usual construction of a random variable which has a given joint law with another, fixed random variable, see Lemma 6.3.

Let us take $\phi_{n} \in \mathcal{A}^{\prime}, n \in \mathbb{N}$ such that

$$
V\left(X_{1}\left(\phi_{n}\right)-W\right) \rightarrow \sup _{\phi \in \mathcal{A}^{\prime}} V\left(X_{1}(\phi)-W\right), n \rightarrow \infty .
$$

Recall that $\gamma$ denotes the conjugate number of $\beta$, see Example 2.4 above. We consider the space $L^{\beta}$ as defined in Example 2.4 above, equipped with the weak topology. We intend to use Corollary 2.3, Lemma 2.2 and Theorem 2.1 with the choice $\mathbb{B}:=L^{\gamma}$ (then $\left.\mathbb{B}^{\prime}=L^{\beta}\right)$ and $M:=\mathcal{D}^{m} \times(\mathbb{R} \cup\{-\infty\})$, $\mu_{n}:=\operatorname{Law}\left(\phi_{n}, Y, X_{1}\left(\phi_{n}\right)\right)$.

First we show that $\phi_{n}: \Omega \rightarrow L^{\beta}$ is measurable when $L^{\beta}$ is equipped with the Borel field of the weak topology. It clearly suffices to show that, for all $\mathcal{G}_{1} \otimes \mathcal{B}([0,1])$-measurable $\zeta$ with $\int_{0}^{1}\left|\zeta_{t}(\omega)\right|^{\beta} d t<\infty, \omega \rightarrow \int_{0}^{1} \zeta(t)(\omega) q(t) d t$ is measurable for all $q \in L^{\gamma}$. Approximating $\zeta$ by step functions and using linearity of the integral, it is enough to show this for $\zeta:=1_{K}$ where $K \in$ $\mathcal{G}_{1} \otimes \mathcal{B}([0,1])$. A monotone class argument reduces this to the case where $K=A \times B$ with $A \in \mathcal{G}_{1}$ and $B \in \mathcal{B}([0,1])$. But then

$$
\int_{0}^{1} \zeta(t)(\omega) q(t) d t=1_{A}(\omega) \int_{B} q(t) d t
$$

which is trivially $\mathcal{G}_{1}$-measurable.

$X_{1}\left(\phi_{n}\right)$ is a $(\mathbb{R} \cup\{-\infty\}$-valued) random variable by Lemmata 6.4 and 6.5. Finally, $Y: \Omega \rightarrow \mathcal{D}$ is measurable, see Remark 3.3 above. This clearly implies the measurability of the triplet $\left(\phi_{n}, Y, X_{1}\left(\phi_{n}\right)\right)$.

(6) implies that $\operatorname{Law}\left(X_{1}\left(\phi_{n}\right)\right)$ is a tight sequence in $\mathbb{R} \cup\{-\infty\}$. Clearly,

$$
\inf _{n} V\left(X_{1}\left(\phi_{n}\right)-W\right)>-\infty
$$

so necessarily $\sup _{n} V_{-}\left(\left[X_{1}\left(\phi_{n}\right)-W\right]^{-}\right)<\infty$, by (6) and by $V_{+}\left([B-W]^{+}\right)<$ $\infty$ in Assumption 4.2 .

Lemma 3.12 of [45] (with the choice $s:=1, a:=\delta_{2}, b:=\delta_{1}$ ) implies that also $\sup _{n} E\left(X_{1}\left(\phi_{n}\right)-W\right)^{-}<\infty$. By the proof of Lemma 3.4 of [22] and by 
$E\left(X_{1}\left(\phi_{n}\right)-W\right)^{-} \leq E\left(X_{1}\left(\phi_{n}\right)\right)^{-}+E W^{+}$, we get that

$$
\begin{aligned}
& E \int_{0}^{1}\left|\phi_{n}(t)\right|^{\beta}(1+|S(t)|)^{\beta} d t \leq E\left(X_{1}\left(\phi_{n}\right)\right)^{-}+E W^{+}+ \\
& 2^{\beta /(\alpha-\beta)} E \int_{0}^{1} H^{\beta /(\beta-\alpha)}\left(S_{t}\right)\left(1+\left|S_{t}\right|\right)^{\beta \alpha /(\alpha-\beta)} d t+1=: \quad C<\infty,
\end{aligned}
$$

by Assumptions 3.7 and 4.2. As $C$ is independent of $n$, Markov's inequality implies

$$
P\left(\int_{0}^{1}\left|\phi_{n}(t)\right|^{\beta}(1+|S(t)|)^{\beta} d t \geq r\right) \leq C / r,
$$

for all $r>0$. Noting that closed balls of $L^{\beta}$ around the origin are weakly compact by the Banach-Alaoglu theorem (since $L^{\beta}$ is a reflexive Banach space), we get that $\operatorname{Law}\left(\phi_{n}\right), n \in \mathbb{N}$ is a tight sequence of probabilities on $\mathcal{B}\left(L^{\beta}\right)$. Finally, as $Y$ takes values in a Polish space, $\operatorname{Law}(Y)$ is tight. It follows that $\mu_{n}$ is tight on $\mathcal{B}\left(L^{\beta} \times \mathcal{D}^{m} \times(\mathbb{R} \cup\{-\infty\})\right)$.

Now apply Corollary 2.3, Lemma 2.2 and Theorem 2.1 to get a probability space $(O, \mathcal{H}, Q)$ and $L^{\beta} \times \mathcal{D} \times(\mathbb{R} \cup\{-\infty\})$-valued random variables $\left(\tilde{\phi}_{n}, Y_{n}, X_{n}\right)$ that converge a.s. to $\left(\phi^{*}, Y^{*}, X^{*}\right)$ along a subsequence (for which we keep the same notation) and $\operatorname{Law}_{Q}\left(\tilde{\phi}_{n}, Y_{n}, X_{n}\right)=\operatorname{Law}\left(\phi_{n}, Y, X_{1}\left(\phi_{n}\right)\right)$, $n \in \mathbb{N}$. Passing to a further subsequence, we may and will assume $S_{n}:=$ $f\left(Y_{n}\right) \rightarrow S^{*}:=f\left(Y^{*}\right)$ a.s. in $\mathcal{D}^{1}$, by Lemmata 3.4, 6.2 and by the fact that each $Y_{n}$ has the same law (on $\mathcal{D}^{m}$ ). Analogously, we may and will assume $W_{n}=\ell\left(Y_{n}\right) \rightarrow W^{*}:=\ell\left(Y^{*}\right)$ a.s. in $\mathbb{R}$. By the argument of Lemma 6.6, we may assume that $\tilde{\phi}_{n}$ can be identified with a $\mathcal{H} \otimes \mathcal{B}([0,1])$-measurable process.

Let us define the analogue of the functionals $V_{ \pm}, V$, for real-valued random variables $X$ on $(O, \mathcal{H}, Q)$.

$$
V_{+}^{Q}(X):=\int_{0}^{\infty} w_{+}\left(Q\left(u_{+}(X) \geq y\right)\right) d y
$$

and

$$
V_{-}^{Q}(X):=\int_{0}^{\infty} w_{-}\left(Q\left(u_{-}(X) \geq y\right)\right) d y .
$$

For each $X$ with $V_{-}^{Q}\left(X^{-}\right)<\infty$ we set

$$
V^{Q}(X):=V_{+}^{Q}\left(X^{+}\right)-V_{-}^{Q}\left(X^{-}\right) .
$$

Define

$$
B_{n}:=\int_{0}^{1} \frac{\alpha-1}{\alpha} \alpha^{1 /(1-\alpha)} H^{1 /(1-\alpha)}\left(S_{t}^{n}\right)\left|S_{t}^{n}\right|^{\alpha /(\alpha-1)} d t
$$


see (5) and (6). The family $\left(B_{n}, W_{n}\right), n \in \mathbb{N}$ has the same law under $Q$ (that of $(B, W)$ under $P)$ hence the family of functions

$$
t \rightarrow w_{+}\left(Q\left(u_{+}\left(\left[B_{n}-W_{n}\right]^{+}\right) \geq t\right)\right), n \in \mathbb{N},
$$

is uniformly integrable (with respect to the Lebesgue measure on $\mathbb{R}_{+}$), by Assumption 4.2. As for all $n$,

$$
-\int_{0}^{1} \tilde{\phi}_{n}(t) S_{n}(t) d t-\int_{0}^{1} H\left(S_{n}(t)\right)\left|\tilde{\phi}_{n}(t)\right|^{\alpha} d t \leq B_{n} \text { a.s. }
$$

uniform integrability of (10) and Fatou's lemma imply that

$$
V^{Q}\left(X^{*}-W^{*}\right) \geq \limsup _{n} V^{Q}\left(X_{n}-W_{n}\right)
$$

so $V^{Q}\left(X^{*}-W^{*}\right) \geq \sup _{\phi \in \mathcal{A}^{\prime}} V\left(X_{1}(\phi)-W\right)$, in particular, $V^{Q}\left(X^{*}-W^{*}\right)>$ $-\infty$.

With the functional $F$ defined in Lemma 6.4, we have, a.s., $\int_{0}^{1} \tilde{\phi}_{n}(t) S_{n}(t) d t=$ $F\left(\tilde{\phi}_{n}, S_{n}\right)$ and hence, by Lemma 6.4,

$$
\int_{0}^{1} \phi^{*}(t) S^{*}(t) d t=\lim _{n} \int_{0}^{1} \tilde{\phi}_{n}(t) S_{n}(t) d t .
$$

It is also clear that

$$
\int_{0}^{1} \phi^{*}(t) d t=0
$$

since $\tilde{\phi}_{n}$ tends to $\phi^{*}$ a.s. weakly in $L^{\beta}$.

From the almost sure convergence of $\tilde{\phi}_{n}$ to $\phi^{*}$ we get that, for almost every $\omega \in O$,

$$
\sup _{n} \int_{0}^{1}\left|\tilde{\phi}_{n}(t)(\omega)\right|^{\beta} d t<\infty
$$

(since a weakly convergent sequence in $L^{\beta}$ is weakly bounded hence also norm-bounded). A fortiori, $\sup _{n} \int_{0}^{1}\left|\tilde{\phi}_{n}(t)(\omega)\right| d t<\infty$ a.s.

Applying Lemma 6.8 on the probability space $(O \times[0,1], \mathcal{H} \otimes \mathcal{B}([0,1]), Q \otimes$ $L e b)$ we get a subsequence (still denoted by $n$ ) such that $\widehat{\phi}_{N}:=\frac{1}{N} \sum_{n=1}^{N} \tilde{\phi}_{n}$ converge to some $\phi^{\circ} P \otimes L e b$-a.s., $N \rightarrow \infty$, and, by (13), also almost surely in the norm of $L^{1}$. This implies convergence in the weak topology of $L^{1}$. As $\tilde{\phi}_{n}$ and hence also $\widehat{\phi}_{n}$ converge to $\phi^{*}$ in the weak topology of $L^{\beta}$ and thus also in the weak topology of $L^{1}$, we get that $\phi^{\circ}=\phi^{*}$ necessarily, $P \otimes L e b$-a.s. hence we may and will use $\phi^{\circ}$ as a version of $\phi^{*}$ in what follows; $\phi^{\circ}$ is an $\mathcal{H} \times \mathcal{B}([0,1])$-measurable process. 
Continuity of $H$ implies $H\left(S_{n}(t)\right) \rightarrow H\left(S^{*}(t)\right)>0$ a.s. so Fatou's lemma and convexity of $x \rightarrow|x|^{\alpha}$ lead to

$$
\begin{array}{r}
-X^{*}-\int_{0}^{1} S^{*}(t) \phi^{*}(t) d t= \\
\lim _{N} \frac{1}{N} \sum_{n=1}^{N} \int_{0}^{1} H\left(S_{n}(t)\right)\left|\tilde{\phi}_{n}(t)\right|^{\alpha} d t \geq \\
\int_{0}^{1} \liminf _{N} \frac{1}{N} \sum_{n=1}^{N} H\left(S_{n}(t)\right)\left|\tilde{\phi}_{n}(t)\right|^{\alpha} d t= \\
\int_{0}^{1} \liminf _{N} \frac{1}{N} \sum_{n=1}^{N} H\left(S^{*}(t)\right)\left|\tilde{\phi}_{n}(t)\right|^{\alpha} d t \geq \\
\int_{0}^{1} H\left(S^{*}(t)\right) \liminf _{N}\left|\widehat{\phi}_{N}(t)\right|^{\alpha} d t= \\
\int_{0}^{1} H\left(S^{*}(t)\right)\left|\phi^{*}(t)\right|^{\alpha} d t .
\end{array}
$$

It follows that $-\int_{0}^{1} S^{*}(t) \phi^{*}(t) d t-\int_{0}^{1} H\left(S^{*}(t)\right)\left|\phi^{*}(t)\right|^{\alpha} d t \geq X^{*}$ so

$V^{Q}\left(-\int_{0}^{1} S^{*}(t) \phi^{*}(t) d t-\int_{0}^{1} H\left(S^{*}(t)\right)\left|\phi^{*}(t)\right|^{\alpha} d t-W^{*}\right) \geq \sup _{\phi \in \mathcal{A}^{\prime}} V\left(X_{1}(\phi)-W\right)$.

Let us invoke Lemma 6.3 with the choice $\tilde{\phi}:=\phi^{*}, \tilde{H}:=Y^{*}$ and $H:=$ $Y$. We get a $\mathcal{G}_{1}$-measurable random element $\phi^{\ddagger}:=\phi \in L^{\beta}$ satisfying $\operatorname{Law}\left(\phi^{\ddagger}, Y\right)=\operatorname{Law}_{Q}\left(\phi^{*}, Y^{*}\right)$. Let us fix $0 \leq t<u \leq 1$. We recall that $\phi_{n} 1_{[0, t]}$ is independent from $Y(u)-Y(t)$, or equivalently,

$$
\operatorname{Law}\left(\phi_{n} 1_{[0, t]}, Y(u)-Y(t)\right)=\operatorname{Law}\left(\phi_{n} 1_{[0, t]}\right) \otimes \operatorname{Law}(Y(u)-Y(t)) .
$$

By construction, $\operatorname{Law}\left(\phi_{n} 1_{[0, t]}, Y(u)-Y(t)\right)=\operatorname{Law}_{Q}\left(\tilde{\phi}_{n} 1_{[0, t]}, Y_{n}(u)-Y_{n}(t)\right)$. This implies also

$$
\operatorname{Law}_{Q}\left(\tilde{\phi}_{n} 1_{[0, t]}, Y_{n}(u)-Y_{n}(t)\right)=\operatorname{Law}_{Q}\left(\tilde{\phi}_{n} 1_{[0, t]}\right) \otimes \operatorname{Law}_{Q}\left(Y_{n}(u)-Y_{n}(t)\right) .
$$

Passing to the limit as $n \rightarrow \infty$,

$$
\operatorname{Law}_{Q}\left(\phi^{*} 1_{[0, t]}, Y^{*}(u)-Y^{*}(t)\right)=\operatorname{Law}_{Q}\left(\phi^{*} 1_{[0, t]}\right) \otimes \operatorname{Law}_{Q}\left(Y^{*}(u)-Y^{*}(t)\right),
$$

which implies independence of $\phi^{\ddagger} 1_{[0, t]} \in L^{\beta}$ from ${ }_{t} Y \in \mathcal{D}^{m}$ as well where $\left({ }_{t} Y\right)_{s}:=0$ if $0 \leq s \leq t$ and $\left({ }_{t} Y\right)_{s}:=Y_{s}-Y_{t}, t<s \leq 1$. 
Since $Y$ is clearly a measurable function of $\left({ }_{t} Y,{ }^{t} Y\right) \in \mathcal{D}^{m} \times \mathcal{D}^{m}$, applying Lemma 6.1 with the choice $\mathfrak{b}:={ }_{t} Y$ and $\mathfrak{a}:=\left(U,{ }^{t} Y\right)$ we get that $\phi^{\ddagger} 1_{[0, t]}$ is $\mathcal{G}_{t^{-}}$ measurable, for all $t$, see Remark 3.3. Applied to $\phi:=\phi^{\ddagger}$, Lemma 6.6 provides a $\mathcal{G}_{t}$-progressively measurable $\phi^{\dagger}$ such that $\operatorname{Law}_{Q}\left(\phi^{*}, Y^{*}\right)=\operatorname{Law}\left(\phi^{\dagger}, Y\right)$, so $\phi^{\dagger} \in \mathcal{A}$.

$\operatorname{As} \operatorname{Law}\left(\phi^{\dagger}, Y\right)=\operatorname{Law}_{Q}\left(\phi^{*}, Y^{*}\right)$, also $\operatorname{Law}\left(\phi^{\dagger}, S\right)=\operatorname{Law}_{Q}\left(\phi^{*}, S^{*}\right)$. Recalling Lemmata 6.4 and 6.5 ,

$\operatorname{Law}_{Q}\left(-\int_{0}^{1} S^{*}(t) \phi^{*}(t) d t-\int_{0}^{1} H\left(S^{*}(t)\right)\left|\phi^{*}(t)\right|^{\alpha} d t-W^{*}\right)=\operatorname{Law}\left(X_{1}\left(\phi^{\dagger}\right)-W\right)$.

It follows from (14) that $V\left(X_{1}\left(\phi^{\dagger}\right)-W\right)>-\infty$ and $\int_{0}^{1} \phi^{\dagger}(t) d t=0$ by (12) hence $\phi^{\dagger} \in \mathcal{A}^{\prime}$ and $\phi^{\dagger}$ is the maximizer we have been looking for.

Remark 4.6. The last part of the proof shows why the independent increments property of $Y$ is a crucial hypothesis. Even if the sequence $\phi_{n} 1_{[0, t]}$ is $\mathcal{G}_{t}$-measurable this does not necessarily hold for its limit (in any sense). So we proceed by noticing that $\tilde{\phi}_{n} 1_{[0, t]}$ are "orthogonal" to ${ }_{t} Y_{n}$ and this property easily passes to the limit and leads to the eventual construction of $\phi^{\dagger}$.

The need for $U$ is also apparent: taking a limit in weak convergence may easily generate additional randomness (think about the construction of weak solutions for stochastic equations such as the Tanaka equation) and hence $\phi^{*} 1_{[0, t]}$ is not necessarily a functional of $Y^{*}$ (even though each $\tilde{\phi}_{n}$ was a functional of $Y_{n}$ ).

Remark 4.7. It is worth commenting on the use of convex combinations in papers dealing with concave utility functions (e.g. [35, 31, 53]) in comparison with the current paper.

When the utility function is concave and no distortions are present then using convex combinations improves performance (either in the utility maximization or in its dual problem where minimization of a convex functional is considered). Converging convex combinations thus directly yield an optimizer in these cases.

The present setting is essentially different: the optimizer is found as the weak limit of a sequence of laws. At this point, taking convex combinations $\widehat{\phi}_{n}$ of the $\phi_{n}$ would not make sense since, by lack of convexity, $v_{n}:=V\left(X_{1}\left(\widehat{\phi}_{n}\right)-W\right)$ will not necessarily dominate the respective convex combintions of $V\left(X_{1}\left(\phi_{n}\right)-W\right)$ and thus $v_{n}$ may cease to converge to $\sup _{\phi} V\left(X_{1}(\phi)-W\right)$.

In our approach, convex combinations are needed at a subsequent stage, in order to show that the $X^{*}$ we constructed is indeed (dominated by) a portfolio value. The representation of Theorem 2.1 is crucial in that argument as it 
permits to form such convex combinations on the auxiliary probability space $(O, \mathcal{H}, Q)$. A similar use of Skorohod's representation theorem appears in the proof of Lemma A.6.4 in [29] where we drew our inspiration from.

Remark 4.8. Theorem 4.5 proves the existence of an optimizer in the family of randomized strategies, i.e. $\mathcal{G}_{t}$-progressively measurable ones. Such a formulation with "relaxed" controls is standard, see e.g. [21, 9], but there are at least two additional arguments in favour of this family in our specific setting.

Lack of concavity and the presence of distortions seem to exclude arguments based on almost sure convergence of convex combinations (e.g. the Komlós lemma, see Lemma 6.7) which is the typical technique for existence proofs in infinite dimensional spaces, see e.g. [35, 19]. The natural way to attack such problems is switching to convergence in law (as usual in the weak formulation of stochastic differential equations, too), see e.g. [15, 14]. However, it was demonstrated in Section 5 of 44 that the set of laws of attainable portfolio values can easily fail to be closed for weak convergence, even in one-step, frictionless models. Finding an optimizer over a non-closed domain looks hopeless. It was shown in Section 6 of [14] in a discrete-time setting that the set of attainable portfolio values becomes closed when using randomized strategies. This is the first reason for our choice of the class of feasible strategies.

As noticed in Section 5 of [14, the investor may actually increase her satisfaction by randomizing her strategy, a phenomenon due to the presence of distortions. This is a second argument for the use of $\mathcal{G}_{t}$-adapted strategies.

It is a delicate question under what kind of conditions Theorem 4.5 remains true with $\mathcal{G}_{t}$-measurable strategies replaced by $\mathcal{F}_{t}$-measurable ones. This is object of current research.

Remark 4.9. It would also be desirable to exhibit cases where optimal strategies can be found that are adapted to $\left(\mathcal{F}_{t}^{S}\right)_{t \in[0,1]}$, the filtration generated by the asset price $S$. As explained in Remark 4.7, it doesn't seem possible to carry out the construction of optimal strategies using almost sure convergence, so even if we start with an $\left(\mathcal{F}_{t}^{S}\right)_{t \in[0,1] \text {-adapted optimizer }}$ sequence there is no guarantee that an $\left(\mathcal{F}_{t}^{S}\right)_{t \in[0,1]}$-adapted limit could be found.

One could expect positive results in the case where $\left(\mathcal{F}_{t}^{S}\right)_{t \in[0,1]}$ is "rich enough" in the sense that for every

$$
\mu \in\left\{\operatorname{Law}\left(X_{1}(\phi)\right): \phi \in \mathcal{A}^{\prime}\right\}
$$

there exists $\lambda$, adapted to $\left(\mathcal{F}_{t}^{S}\right)_{t \in[0,1]}$, such that $\operatorname{Law}\left(X_{1}(\lambda)\right)=\mu$. This seems 
to require delicate arguments even in the case of frictionless markets and hence lies beyond the scope of the present work.

Remark 4.10. The main lines of the above proof seem to work in situations with model uncertainty as well. In that case $V\left(X_{1}(\phi)\right)=V^{S}\left(X_{1}(\phi)\right)$ is calculated for each $S \in \mathcal{S}$ where $\mathcal{S}$ is a family of price processes and one tries to maximize the worst-case functional $\inf _{S \in \mathcal{S}} V^{S}\left(X_{1}(\phi)\right)$ over $\phi$, see e.g. [38, 37].

As an optimizing sequence one can consider $\left(\phi^{n}, S_{n}\right)$ instead of $\left(\phi^{n}, Y_{n}\right)$ in this case (assuming e.g. that each $S \in \mathcal{S}$ generates the same filtration as $Y$ ). In this setting the $S_{n}$ may well have different laws. Nevertheless, under appropriate tightness conditions the construction above may provide an optimizer $\phi^{*}$ as well as the worst-case model $S^{*}=\operatorname{argmin}_{S} V^{S}\left(X_{1}\left(\phi^{*}\right)\right)$. These ideas are left for exploration in future research.

\section{A formulation with generalized strategies}

In the theory of stochastic differential equations, the concept of weak solutions allows to vary the underlying probability space, giving more flexibility for the construction of solutions. Weak convergence of probability measures and Skorohod's theorem are typical tools in that area.

As weak convergence techniques predominate in the present paper as well and the solution $\phi^{\dagger}$ is also a "weak" one, in this section we reformulate problem (4) in a manner that is closer in spirit to the world of weak solutions.

Definition 5.1. Let $\left(\Omega, \mathcal{F},\left(\mathcal{F}_{t}\right)_{t \in[0,1]}, P\right), Y, S, W$ be as in Section 3. A fivetuple

$$
\pi=(O, \mathcal{H}, Q, \tilde{Y}, \tilde{\phi})
$$

is called a generalized strategy if $(O, \mathcal{H}, Q)$ is a probability space, $\tilde{Y}_{t}, t \in[0,1]$ is a càdlàg process on $(O, \mathcal{H}, Q)$ identical in law to $Y$, and $\tilde{\phi}$ is a process on $(O, \mathcal{H}, Q)$ such that $\tilde{\phi}_{s}$ is independent of $\tilde{Y}_{u}-\tilde{Y}_{t}$ for all $0 \leq s \leq t<u$ and $\int_{0}^{1}\left|\tilde{\phi}_{t}\right| d t<\infty$ Q-a.s.

Clearly, $\tilde{Y}$ is the copy of $Y$ providing the information structure on $(O, \mathcal{H}, Q)$ and $\tilde{\phi}$ represents the trading speed which must be non-anticipative with respect to $\tilde{Y}$. Defining $\tilde{W}:=\ell(\tilde{Y})$ and $\tilde{S}:=f(\tilde{Y})$, we can set

$$
\begin{aligned}
X_{1}(\tilde{\phi}) & :=-\int_{0}^{1} \tilde{\phi}_{t} \tilde{S}_{t} d t-\int_{0}^{1} H\left(\tilde{S}_{t}\right)\left|\tilde{\phi}_{t}\right|^{\alpha} d t \\
\tilde{X}_{1}(\tilde{\phi}) & :=\int_{0}^{1} \tilde{\phi}_{t} d t .
\end{aligned}
$$


Let $\Pi$ denote the class of generalized strategies. Another version of Theorem 4.5 could be stated as follows. Its proof closely follows that of Theorem 4.5 .

Theorem 5.2. Let Assumptions 3.1, 3.7 and 4.2 be in force. Let $\Pi^{\prime}:=\{\pi \in$ $\left.\Pi: V_{-}^{Q}\left(\left[X_{1}(\tilde{\phi})-\tilde{W}\right]^{-}\right)<\infty, \tilde{X}_{1}(\phi)=0\right\}$. There exists $\pi^{\dagger} \in \Pi^{\prime}$ such that

$$
V^{Q^{\dagger}}\left(X_{1}\left(\tilde{\phi}^{\dagger}\right)-W^{\dagger}\right)=\sup _{\pi \in \Pi^{\prime}} V^{Q}\left(X_{1}(\tilde{\phi})-\tilde{W}\right)
$$

where $\pi^{\dagger}=\left(O^{\dagger}, \mathcal{H}^{\dagger}, Q^{\dagger}, \tilde{Y}^{\dagger}, \tilde{\phi}^{\dagger}\right)$ and $W^{\dagger}=\ell\left(\tilde{Y}^{\dagger}\right)$.

\section{$6 \quad$ Auxiliary results}

Lemma 6.1. Let $(A, \mathcal{A}),(B, \mathcal{B})$ be measurable spaces and $j: A \times B \rightarrow \mathbb{R}$ a measurable mapping. Let $(\mathfrak{a}, \mathfrak{b})$ be an $A \times B$-valued random variable. If $\sigma(j(\mathfrak{a}, \mathfrak{b}), \mathfrak{a})$ is independent of $\mathfrak{b}$ then $j(\mathfrak{a}, \mathfrak{b})$ is $\sigma(\mathfrak{a})$-measurable.

Proof. Denote by $\mu_{A}(\cdot)$ (resp. $\left.\mu_{B}(\cdot)\right)$ the law of $\mathfrak{a}$ (resp. $\mathfrak{b}$ ). Considering arctan $\circ j$ instead of $j$, we may and will assume that $j$ is bounded. We claim that $j(\mathfrak{a}, \mathfrak{b})=k(\mathfrak{b})$ a.s. where $k(a):=\int_{B} j(a, b) \mu_{B}(d b)$. Using an argument with monotone classes, it suffices to establish that, for all bounded measurable $m: A \rightarrow \mathbb{R}, n: B \rightarrow \mathbb{R}$, one has $\operatorname{Ej}(\mathfrak{a}, \mathfrak{b}) m(\mathfrak{a}) n(\mathfrak{b})=\operatorname{Ek}(\mathfrak{a}) m(\mathfrak{a}) n(\mathfrak{b})$. By independence of $\mathfrak{a}, \mathfrak{b}$ and by definition, the latter expression equals

$$
\begin{gathered}
\operatorname{Ek}(\mathfrak{a}) m(\mathfrak{a}) \operatorname{En}(\mathfrak{b})=\int_{A} \int_{B} j(a, w) \mu_{B}(d w) m(a) \mu_{A}(d a) \operatorname{En}(\mathfrak{b})= \\
\int_{A} \int_{B} j(a, w) m(a) \mu_{B}(d w) \mu_{A}(d a) \operatorname{En}(\mathfrak{b})=\operatorname{Ej}(\mathfrak{a}, \mathfrak{b}) m(\mathfrak{a}) \operatorname{En}(\mathfrak{b})= \\
\operatorname{Ej}(\mathfrak{a}, \mathfrak{b}) m(\mathfrak{a}) n(\mathfrak{b}),
\end{gathered}
$$

by our independence hypothesis. This completes the proof.

We now recall Théorème 1 of [3]. Just like Theorem 2.1] in Section [2] above, this result is crucial for the developments of the present paper.

Lemma 6.2. Let $A, B$ be separable metric spaces and $\xi_{n} \in A, n \in \mathbb{N} a$ sequence of random variables converging to $\xi \in A$ in probability such that Law $\left(\xi_{n}\right)$ is the same for all $n$. Then for each measurable $h: A \rightarrow B$ the random variables $h\left(\xi_{n}\right)$ converge to $h(\xi)$ in probability (hence also a.s. along a subsequence). 
Lemma 6.3. Let the topological space $Z$ be the union of its closed, increasing subspaces $A_{n}, n \in \mathbb{N}$ which are Polish spaces (with appropriate metrics) and let $B$ be a measurable space. Let $H, \tilde{H}$ be random elements in $B$ with identical laws, defined on the probability spaces $(\Xi, \mathcal{E}, R),(\tilde{\Xi}, \tilde{\mathcal{E}}, \tilde{R})$, respectively. Let $\tilde{\phi}$ be a random element in $Z$, defined on $(\tilde{\Xi}, \tilde{\mathcal{E}}, \tilde{R})$. Let $U$ be independent of $H$ with uniform law on $[0,1]$. There exists a measurable function $f: B \times[0,1] \rightarrow$ $Z$ such that $\phi=f(H, U)$ satisfies $\operatorname{Law}_{R}(H, \phi)=\operatorname{Law}_{\tilde{R}}(\tilde{H}, \tilde{\phi})$.

Proof. In view of Lemma 3.22 of 33 it suffices to show that $Z$ is a Borel space in the sense of [33], i.e. it is Borel-isomorphic to a Borel subset of $[0,1]$. We define $C_{n}:=A_{n} \backslash \cup_{i<n} A_{i}, n \geq 0$. Borel subsets of Polish spaces are clearly Borel spaces, let $\psi_{n}: C_{n} \rightarrow\left[1-1 / 2^{2 n+1}, 1-1 / 2^{2 n+2}\right]$ be Borel isomorphisms attesting this. Then it is easy to check that $\psi(x):=\psi_{n}(x), x \in C_{n}$ defines a Borel isomorphism between $Z$ and a Borel subset of $[0,1]$.

Lemma 6.4. The mapping $F: L^{\beta} \times \mathcal{D}^{1} \rightarrow \mathbb{R}$ defined by $F(\psi, \chi):=\int_{[0,1]} \psi(t) \chi(t) d t$ is sequentially continuous and (jointly) measurable when $L^{\beta}$ is equipped with the weak topology.

Proof. Take sequences $\psi_{n} \rightarrow \psi$ in $L^{\beta}$ and $\chi_{n} \rightarrow \chi$ in $\mathcal{D}^{1}$. Then the sequence $\chi_{n}$, being relatively compact in $\mathcal{D}^{1}$, is uniformly bounded by a constant $K$ (see Theorem 12.3 of [8]) and $\chi_{n}(t)$ tends to $\chi(t)$ at every continuity point $t$ of the latter, in particular outside a countable set (see page 124 of [8]). So

$$
\begin{array}{r}
\left|\int_{[0,1]} \psi_{n}(t) \chi_{n}(t) d t-\int_{[0,1]} \psi(t) \chi(t) d t\right| \leq \\
\int_{[0,1]}\left|\psi_{n}(t) \chi_{n}(t)-\psi_{n}(t) \chi(t)\right| d t+\int_{[0,1]}\left|\psi_{n}(t) \chi(t)-\psi(t) \chi(t)\right| d t \leq \\
\left(\int_{[0,1]}\left|\psi_{n}(t)\right|^{\beta} d t\right)^{1 / \beta}\left(\int_{[0,1]}\left|\chi_{n}(t)-\chi(t)\right|^{\gamma} d t\right)^{1 / \gamma}+ \\
\int_{[0,1]}\left|\psi_{n}(t) \chi(t)-\psi(t) \chi(t)\right| d t
\end{array}
$$

The first term tends to 0 as $\psi_{n}$ is weakly bounded hence also norm bounded in $L^{\beta}$ and Lebesgue's theorem applies to $\left|\chi_{n}(t)-\chi(t)\right|^{\gamma} \leq(2 K)^{\gamma}$. The second term tends to 0 by the weak convergence of $\psi_{n}$ to $\psi$ noting that $\chi \in L^{\gamma}$ trivially.

As closed balls with radius $r$ around the origin in $L^{\beta}$ (denoted by $B_{r}$ ) are metrizable by the separability of $L^{\gamma}$, sequential continuity implies continuity and hence measurability of $F$ restricted to $B_{r} \times \mathcal{D}^{1}$ for every $r$, which easily implies the measurability of $F$ on the whole of $L^{\beta} \times \mathcal{D}^{1}$. 
Lemma 6.5. The mapping $(s, \phi) \in \mathcal{D}^{1} \times L^{\beta} \rightarrow \int_{0}^{1} H(s(t))|\phi(t)|^{\alpha} d t \in \mathbb{R}$ is $\mathcal{B}\left(\mathcal{D}^{1} \times L^{\beta}\right)$ measurable when $L^{\beta}$ is equipped by the weak topology.

Proof. By the monotone convergence theorem it is enough to prove the measurability of

$$
(s, \phi) \rightarrow \int_{0}^{1} H(s(t))\left(|\phi(t)|^{\alpha} \wedge N\right) d t
$$

for all $N>0$. Since $L^{\beta}$ is a separable Banach space, by results of [42], Borel sets of $L^{\beta}$ for the weak topology coincide with those of the norm topology. So it suffices to prove continuity of (15) when $L^{\beta}$ is equipped with the norm topology. Let $\left(s_{n}, \phi_{n}\right) \rightarrow(s, \phi)$ in $\mathcal{D}^{1} \times L^{\beta}$. Then $s_{n}$ are uniformly bounded and converge Lebesgue-a.s. to $s$ and $\phi_{n}$ converge to $\phi$ in Lebesgue measure. Dominated convergence implies the convergence of $\int_{0}^{1} H\left(s_{n}(t)\right)\left(\left|\phi_{n}(t)\right|^{\alpha} \wedge\right.$ $N) d t$ to $\int_{0}^{1} H(s(t))\left(|\phi(t)|^{\alpha} \wedge N\right) d t$ as $n \rightarrow \infty$.

Lemma 6.6. Let $\phi: \Omega \rightarrow L^{\beta}$ be such that $\sigma\left(\phi 1_{[0, t]}\right) \subset \mathcal{G}_{t}$ for all $t$. Then there exists $\bar{\phi}(\omega, t)=\phi(\omega)(t), P \times$ Leb-a.s. such that $\bar{\phi}_{t}$ is $\mathcal{G}_{t}$-progressively measurable.

Proof. Define

$$
\check{\phi}(\omega, t):=\limsup _{n} n \int_{t-1 / n}^{t} \phi(\omega)(s) d s, \quad \bar{\phi}(\omega, t):=\check{\phi}(\omega, t) 1_{\{\check{\phi}(\omega, t)<\infty\}} .
$$

By Lebesgue's differentiation theorem and by measurability of $\omega \rightarrow \phi(\omega) \in$ $L^{\beta}$ this is $\mathcal{F} \otimes \mathcal{B}([0,1])$-measurable and equals $\phi(\omega)(t), P \otimes L e b$-a.s. By $\sigma\left(\phi 1_{[0, t]}\right) \subset \mathcal{G}_{t}$ we get progressive measurability, too.

We recall the main result of [34, see the Appendix of [29] for a recent account of the proof.

Lemma 6.7. Let $f_{n}$ be a sequence of real-valued random variables satisfying

$$
\sup _{n} E\left|f_{n}\right|<\infty
$$

Then there is a subsequence $n_{j}, j \in \mathbb{N}$ and a random variable $f$ such that

$$
\widehat{f_{i}}:=\frac{1}{i} \sum_{j=1}^{i} f_{n_{j}} \rightarrow f, \text { a.s., } i \rightarrow \infty .
$$

We will need an easy corollary of the above lemma, used in the proof of Theorem 4.5. 
Corollary 6.8. Let $f_{n}: O \times[0,1] \rightarrow \mathbb{R}, n \in \mathbb{N}$ be $\mathcal{H} \otimes \mathcal{B}([0,1])$-measurable such that

$$
J:=\sup _{n} \int_{0}^{1}\left|f_{n}(\omega, t)\right| d t<\infty
$$

almost surely. Then there is a subsequence $n_{j}, j \in \mathbb{N}$ and $f: O \times[0,1] \rightarrow \mathbb{R}$ such that

$$
\widehat{f_{i}}:=\frac{1}{i} \sum_{j=1}^{i} f_{n_{j}} \rightarrow f, Q \otimes \text { Leb-a.s., } i \rightarrow \infty .
$$

Proof. Define $d \mu / d(Q \otimes L e b):=e^{-J} / E e^{-J}$. Under $\mu$, Lemma 6.7 applies to the sequence $f_{n}$ so we get $\widehat{f}_{i}$ converging to $f, \mu$-a.s. Since $\mu \sim Q \otimes L e b$, this completes the proof.

\section{Acknowledgments}

The authors thank the anonymous referees for their useful comments and gratefully acknowledge the support of the "Lendület" grant LP 2015-6 of the Hungarian Academy of Sciences.

\section{References}

[1] R. Almgren And N. Chriss, Optimal execution of portfolio transactions, Journal of Risk, 3 (2001), pp. 5-40.

[2] T. O. Banakh, V. I. Bogachev, And A. Kolesnikov, Topological spaces with Skorokhod representation property, Ukrainian Mathematical Journal, 57 (2005), pp. 1371-1386.

[3] M. T. Barlow, M. Émery, F. B. Knight, S. Song, And M. Yor, Autour d'un théorème de tsirelson sur des filtrations browniennes et non browniennes, in Séminaire de Probabilités XXXII, Springer, 1998, pp. 264-305.

[4] A. B. Berkelana, R. Kouwenberg, and T. Post, Optimal portfolio choice under loss aversion, Review of Economics and Statistics, 86 (2004), pp. 973-987.

[5] C. Bernard and M. Ghossoub, Static portfolio choice under cumulative prospect theory, Mathematics and Financial Economics, 2 (2010), pp. 277-306. 
[6] S. Biagini AND M. Frittelli, Utility maximization in incomplete markets for unbounded processes, Finance and Stochastics, 9 (2005), pp. $493-517$.

[7] S. Biagini and M. Frittelli, A unified framework for utility maximization problems: an orlicz space approach, Annals of Applied Probability, 18 (2008), pp. 929-966.

[8] P. Billingsley, Convergence of probability measures, John Wiley \& Sons, 2nd ed., 1999.

[9] V. S. Borkar, Controlled diffusion processes, Probability surveys, 2 (2005), pp. 213-244.

[10] B. BouchaRD, Utility maximization on the real line under proportional transaction costs, Finance and Stochastics, 6 (2002), pp. 495-516.

[11] L. Campi And M. Del Vigna, Weak insider trading and behavioral finance, SIAM Journal on Financial Mathematics, 3 (2012), pp. 242-279.

[12] L. CAmpi And M. P. Owen, Multivariate utility maximization with proportional transaction costs, Finance and Stochastics, 15 (2011), pp. 461-499.

[13] L. Carassus and H. Pham, Portfolio optimization for nonconvex criteria functions, RIMS Kôkyuroku series, ed. Shigeyoshi Ogawa, 1620 (2009), pp. 81-111.

[14] L. Carassus And M. RÁsonyi, On optimal investment for a behavioral investor in multiperiod incomplete market models, Mathematical Finance, 25 (2015), pp. 115-153.

[15] G. Carlier And R.-A. Dana, Optimal demand for contingent claims when agents have law invariant utilities, Mathematical Finance, 21 (2011), pp. 169-201.

[16] H. Chang, J. Cvitanić, And X. Y. Zhou, Optimal contracting with moral hazard and behavioral preferences, Journal of Mathematical Analysis and Applications, 428 (2015), pp. 959-981.

[17] A. Cherny and D. Madan, New measures for performance evaluation, Review of Financial Studies, 22 (2009), pp. 2571-2606.

[18] R. Cont, A. Kukanov, and S. Stoikov, The price impact of order book events, Journal of Financial Econometrics, 12 (2014), pp. 47-88. 
[19] C. Czichowsky and W. Schachermayer, Portfolio optimisation beyond semimartingales: shadow prices and fractional brownian motion, To appear in Annals of Applied Probability, (2016).

[20] Y. Dolinsky And H. M. Soner, Duality and convergence for binomial markets with friction, Finance and Stochastics, 17 (2013), pp. 447-475.

[21] W. H. Fleming, Generalized solutions in optimal stochastic control, in : Differential Games and Control Theory III (eds. E. Roxin, P. T. Liu and R. Sternberg), 1977, pp. 147-165.

[22] P. Guasoni And M. RÁsonyi, Hedging, arbitrage and optimality with superlinear frictions, The Annals of Applied Probability, 25 (2015), pp. 2066-2095.

[23] X. D. He, S. Hu, J. ObŁós, And X. Y. Zhou, Optimal exit time from casino gambling: Strategies of pre-committed and naive gamblers, Submitted, (2017).

[24] X. D. He, S. Hu, J. ObєóJ, And X. Y. Zhou, Path-dependent and randomized strategies in barberis' casino gambling model, To appear in Operations Research, (2017).

[25] X. D. He AND X. Y. ZHOU, Portfolio choice under cumulative prospect theory: An analytical treatment, Management Science, 57 (2011), pp. 315-331.

[26] A. JAkubowski, The almost sure Skorohod representation for subsequences in nonmetric spaces, Theory of Probability \& Its Applications, 42 (1997), pp. 167-175.

[27] H. Jin AND X. Y. ZHOU, Behavioral portfolio selection in continuous time, Mathematical Finance, 18 (2008), pp. 385-426.

[28] H. Jin And X. Y. Zhou, Greed, leverage, and potential losses: A prospect theory perspective, Mathematical Finance, 23 (2013), pp. 122142.

[29] Y. Kabanov and S. Pergamenshchikov, Two-Scale Stochastic Systems, Springer, 2003.

[30] Y. KABANOV AND M. SAFARIAN, Markets with transaction costs: Mathematical Theory, Springer Science \& Business Media, 2009. 
[31] Y. M. Kabanov And C. Stricker, On the optimal portfolio for the exponential utility maximization: remarks to the six-author paper, Mathematical Finance, 12 (2002), pp. 125-134.

[32] D. Kahneman and A. Tversky, Prospect theory: An analysis of decision under risk, Econometrica: Journal of the Econometric Society, 47 (1979), pp. 263-291.

[33] O. Kallenberg, Foundations of modern probability, Springer, 2nd ed., 2002.

[34] J. KomLós, A generalization of a problem of Steinhaus, Acta Mathematica Hungarica, 18 (1967), pp. 217-229.

[35] D. Kramkov and W. Schachermayer, The asymptotic elasticity of utility functions and optimal investment in incomplete markets, Annals of Applied Probability, (1999), pp. 904-950.

[36] R. C. Merton, Lifetime portfolio selection under uncertainty: The continuous-time case, The Review of Economics and Statistics, (1969), pp. 247-257.

[37] A. Neufeld And M. Nutz, Robust utility maximization with Lévy processes, arXiv:1502.05920, (2015).

[38] M. Nutz, Utility maximization under model uncertainty in discrete time, published online by Mathematical Finance, (2014).

[39] M. P. OWEN AND G. ŽITKOviĆ, Optimal investment with an unbounded random endowment and utility-based pricing, Mathematical Finance, 19 (2009), pp. 129-159.

[40] T. Penannen, A.-P. Perkkiö, And M. RÁsonyi, Non-convex dynamic programming and optimal investment, To appear in Mathematics and Financial Economics, arXiv:1504.01903, (2015).

[41] T. Pennanen, Optimal investment and contingent claim valuation in illiquid markets, Finance and Stochastics, 18 (2014), pp. 733-754.

[42] B. J. Pettis, On integration in vector spaces, Transactions of the American Mathematical Society, 44 (1938), pp. 277-304.

[43] J. Quiggin, A theory of anticipated utility, Journal of Economic Behavior \& Organization, 3 (1982), pp. 323-343. 
[44] M. RÁsonyi, Optimal investment with bounded above utilities in discrete time markets, SIAM Journal on Financial Mathematics, 6 (2015), pp. 517-529.

[45] M. RÁsonyi And A. M. Rodrigues, Optimal portfolio choice for a behavioural investor in continuous-time markets, Annals of Finance, 9 (2013), pp. 291-318.

[46] M. RÁsonyi And A. M. Rodrigues, Continuous-time portfolio optimisation for a behavioural investor with bounded utility on gains, Electronic Communications in Probability, 19 (2014), pp. 1-13.

[47] M. Rásonyi and J. G. RodríGuez-Villarreal, Optimal investment under behavioural criteria-a dual approach, in : Advances in Mathematics of Finance, eds. A. Palczewski and L. Stettner, Banach Center Publications, 2015, pp. 167-180.

[48] M. Rásonyi and J. G. RodríGuez-Villarreal, Behavioural investors in continuous-time incomplete markets, Theory of Probability and Applications, 60 (2016), pp. 631-646.

[49] M. RÁsonyi And H. SAYit, Sticky processes, local and true martingales, Submitted, arXiv:1509.08280v3, (2017).

[50] C. ReICHLIN, Utility maximization with a given pricing measure when the utility is not necessarily concave, Mathematics and Financial Economics, 7 (2013), pp. 531-556.

[51] C. R. A. Reichlin, Non-concave utility maximization: optimal investment, stability and applications, PhD thesis, ETH Zürich, 2012.

[52] P. A. Samuelson, Lifetime portfolio selection by dynamic stochastic programming, The Review of Economics and Statistics, (1969), pp. 239246.

[53] W. Schachermayer, Optimal investment in incomplete markets when wealth may become negative, Annals of Applied Probability, 11 (2001), pp. 694-734.

[54] A. Tversky and D. Kahneman, Advances in prospect theory: Cumulative representation of uncertainty, Journal of Risk and Uncertainty, 5 (1992), pp. 297-323. 